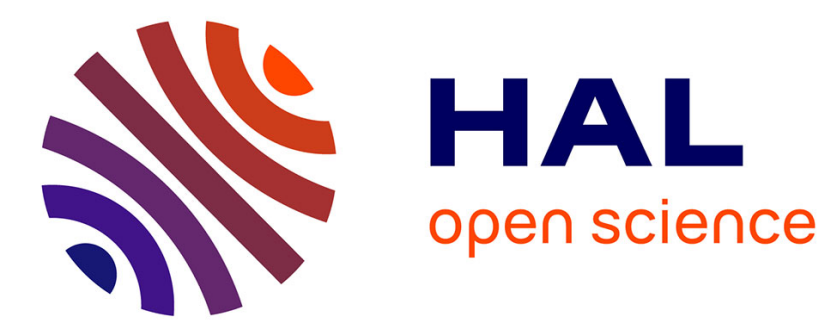

\title{
Persuasive Events and User's Roles in Mobile-Based Interactive Solutions for Nature Discovery
}

\author{
Alessandro Fenicio, Yann Laurillau, Gaëlle Calvary
}

\section{To cite this version:}

Alessandro Fenicio, Yann Laurillau, Gaëlle Calvary. Persuasive Events and User's Roles in MobileBased Interactive Solutions for Nature Discovery. 14th EAI International Conference on Mobile and Ubiquitous Systems: Computing, Networking and Services, Nov 2017, Melbourne, Australia. pp.511$519,10.1145 / 3144457.3145504$. hal-03223719

\section{HAL Id: hal-03223719 \\ https://hal.science/hal-03223719}

Submitted on 13 Jul 2021

HAL is a multi-disciplinary open access archive for the deposit and dissemination of scientific research documents, whether they are published or not. The documents may come from teaching and research institutions in France or abroad, or from public or private research centers.
L'archive ouverte pluridisciplinaire HAL, est destinée au dépôt et à la diffusion de documents scientifiques de niveau recherche, publiés ou non, émanant des établissements d'enseignement et de recherche français ou étrangers, des laboratoires publics ou privés. 


\section{Persuasive Events and User's Roles in Mobile-Based Interactive Solutions for Nature Discovery}

\author{
Alessandro Fenicio \\ Univ. Grenoble Alpes, CNRS, \\ Grenoble INP, LIG, F-38000 Grenoble \\ France \\ Alessandro.Fenicio@ \\ univ-grenoble-alpes.fr
}

\author{
Yann Laurillau \\ Univ. Grenoble Alpes, CNRS, \\ Grenoble INP, LIG, F-38000 Grenoble \\ France \\ Yann.Laurillay@univ-grenoble-alpes.
}

fr

\author{
Gaëlle Calvary \\ Univ. Grenoble Alpes, CNRS, \\ Grenoble INP, LIG, F-38000 Grenoble \\ France \\ Gaelle.Calvary@univ-grenoble-alpes. \\ fr
}

\begin{abstract}
The exploration and discovery of nature through walking, cycling, touring, trekking and hiking are targeted by this work, part of an on-going research on mobile-based persuasive user interaction. Considering $\mathrm{HCI}$ solutions dedicated to discover and explore nature, we uncover the concept of persuasive event and several classes of roles that users may endorse during a long-term usage of such interactive solutions. We claim the importance of these events and roles as, leveraging persuasion, they may contribute to improve nature discovery experience and to sustain motivation through user interaction. In this work we share these insights from our studies on the Mhikes platform, designed for such kind of nature-involved activities.
\end{abstract}

\section{CCS CONCEPTS}

-Human-centered computing $\rightarrow$ User models; Graphical user interfaces; Collaborative interaction; HCI theory, concepts and models; Empirical studies in HCI; Interaction design process and methods; Collaborative and social computing theory, concepts and paradigms; • Applied computing $\rightarrow$ Psychology; Sociology;

\section{KEYWORDS}

Persuasion, Behavioral Change, Persuasive Events, Literature Review, Social Media, User Roles

\section{ACM Reference format:}

Alessandro Fenicio, Yann Laurillau, and Gaëlle Calvary. 2017. Persuasive Events and User's Roles in Mobile-Based Interactive Solutions for Nature Discovery. In Proceedings of the 14th EAI International Conference on Mobile and Ubiquitous Systems: Computing, Networking and Services, Melbourne, VIC, Australia, November 7-10, 2017 (MobiQuitous 2017), 9 pages. https://doi.org/10.1145/3144457.3145504

Permission to make digital or hard copies of all or part of this work for personal or classroom use is granted without fee provided that copies are not made or distributed for profit or commercial advantage and that copies bear this notice and the full citation on the first page. Copyrights for components of this work owned by others than ACM must be honored. Abstracting with credit is permitted. To copy otherwise, or republish, to post on servers or to redistribute to lists, requires prior specific permission and/or a fee. Request permissions from permissions@acm.org.

MobiQuitous 2017, November 7-10, 2017, Melbourne, VIC, Australia

(c) 2017 Association for Computing Machinery.

ACM ISBN 978-1-4503-5368-7/17/11 ..\$15.00

https://doi.org/10.1145/3144457.3145504

\section{INTRODUCTION}

Having your eyes on the mobile phone screen, reading notification messages, or in general, interacting with an electronic device are not the first actions we associate with exploring nature. Naturerelated thematic often involves scenarios of relaxation, meditation or reconciliation between body and mind in a general context of harmonious coexistence with the environment. Technology, nevertheless, has found an important place in nature-related activities, and in particular, HCI has focused on two main perspectives in this domain: augmenting nature with information (for the purpose of learning) and assisting explorers along the way (for navigation).

Mobile-based exploration and discovery of nature through walking, cycling, touring, trekking and hiking are targeted by this work, part of an on-going research on persuasive user interaction. Persuasive technologies aim to support human behavior change without coercion, persuading users to voluntary progress in a process of change [16]. The targeted changes may belong to different domains and impact different aspects in the life of a person. Behavior changes related to health have been widely explored by persuasionrelated studies applied to different domains, for example: quitting smoking [14], quitting drinking [27], diet-related [22] and others.

Cheverst et al. in their work [6] underline how HCI solutions for exploration often focus on the learning purpose of technology. Some examples can be learning types of wood [36] or exploring biodiversity in nature [39]. Other studies focus instead on the collaborative approach, as for example the work of Miura et al. [32]. The work of Rosner et al. [37] takes a different approach on how users could be motivated by exploring an itinerary created and shared by another user. In particular, the path is discovered along the way and it turns into a secret message embedded by the creator.

This shows how distinction between users that create content and users that consume content is implicit: when users create contents they are creators, when they use the contents are consumers. What we suggest is to adapt the HCI solution, and the subsequent design of a mobile application, depending on the role endorsed by the user with dedicated features, to enhance the user experience. In the same way, when the technology (e.g. mobile-phone) is shared among a group of users in a collaborative manner, roles endorsed by users emerge: the role of the users leading the activity (e.g. reading information on the screen) and the role of people following (e.g. relating information on the environment) that do not have dedicated HCI controls/features.

Considering HCI solutions dedicated to discover and explore nature, we uncover several classes of roles that users may endorse during a long-term usage of such interactive solutions. We claim 
the importance of these roles as, leveraging persuasion, they may contribute to increase the nature discovery experience and to sustain motivation through user interaction. Furthermore, we propose the concept of persuasive event as an innovative way to leverage behavior change and to design persuasive technologies supporting collaborative activities. In this work, we identify new persuasive principles to leverage behavior change. To do so, we rely on an analysis grid framed on the transtheoretical model of behavior Change [35] using the methodology proposed by Greenberg et al. [23].

In the following, we begin the article with a selection of background studies on behavior change, persuasion and influence principles. We then characterize four classes of roles and the concept of event on social media platforms to gather the main properties of the current event systems. The central part of this work consists in showing, beside a literature review, persuasive principles that can be applied to events. We provide some examples based on Mhikes platform, a mobile application dedicated to hiking touring and nature discovery, created by the startup Easy Mountain. Easy Mountain is currently maintaining the application improving its features through research and development projects.

In order to illustrate examples of persuasive features associated to events, for each stage of the transtheoretical model of behavior change we provide a context for users, an example on Mhikes platform and a brief discussion.

\section{BEHAVIOR CHANGE, PERSUASION, INFLUENCE}

Research on persuasive technologies is a cross-discipline research field. It is rooted on theories, models and principles borrowed from various disciplines including computer science, social sciences such as psychology, or medicine. Our approach mainly relies on the transtheoretical model but also on other principles such as influence, design techniques and behavior models. This section presents a synthesis of these fundamental concepts.

\subsection{Transtheoretical model of behaviour of change}

The transtheoretical model of behaviour change (TTM) [35] formalizes the steps of the change into a progression that can be achieved using motivation, effort and energy to move among some defined stages. [31]. The transtheoretical model has been used to promote behavior change in a variety of behaviors including: quitting smoking [11], physical activity [28, 29], dietary fat consumption [21] and many others.

The stages progress as follows:

- Precontemplation: where subjects are not considering the idea of change, perhaps because they are unaware or not informed or possibly frustrated by a previous failed change attempt [31]. They do not intend to take action in the next six months [35].

- Contemplation: subjects are aware that they should change a certain behavior [31], and they consider to attempt the change in the next six months [35]. In this stage they try to get informed about the problem, but they are not ready to take a concrete action for changing [31].
- Preparation: subjects are ready to take change in the near future (usually measured as the next month), they are tyring to develop a plan to take their first concrete action in the direction of the change [31].

- Action: subjects are passed to action and modified their behavior within the past six months [35].

- Maintenance: subjects try to keep the behavior change, and struggle to prevent relapsing [35]. In case they fail in this stage a relapse will occur, regress them to an earlier stage and they will have to restart the progress from the first stages [31].

We have presented the transtheoretical model stages but in order to approach the literature review we present some other persuasive principles in the next paragraphs.

\subsection{Behavior Model}

In the Fogg Behaviour Model, persuasion is represented using two dimensions, ability and motivation, plus an enacting trigger. Triggers can be of different types: sparks (used to increase the motivation), signals (which serve only as reminders) and facilitators (used to make the change easier). In this model indeed, the change is likely to happen if the target behavior is sufficiently motivated, it has the ability to perform the behavior, and it is triggered to perform the behavior. These three factors must occur and reach a certain threshold simultaneously to trigger an effective change. Otherwise, the behavior is likely not to happen [18].

Some specific elements can affect the level of ability and motivation. The three core motivators, central to the human experience, are: pleasure/pain, hope/fear and social acceptance/rejection. The factor fostering the ability variable is simplicity since it makes path to an easier change. According to Fogg [18], simplicity consists of six parts (time, money, physical effort, brain cycles, social deviance, and non routine) which are related to each other like links in a chain: if any single link breaks, then the complete chain may fail. In this case, simplicity is lost.

\subsection{Create habits using technology}

According to the studies of Eyal [12] is it possible to design technology in order to form habits. In this perspective an application can start as a "vitamin" (there is no real need for it in the beginning) and then using habit forming techniques can become a "painkiller" (not using it causes pain to the user). He mentions Facebook, Pinterest and Instagram as products that started as simple ideas and have grown exponentially. Eyal evidences four stages in his Hook Model: the trigger, the action, the reward., and the investment.

The trigger stages recalls the ideas of B.J. Fogg as an event that enacts the user [18]. Eyal makes a significant difference between the external triggers (the system tells the user what to do) and the internal trigger (the user knows what to do). Generally internal triggers compensate negative emotions through the product, as for example: compensate loneliness using a social network, looseness using a GPS system and unsureness using a search engine.

The action stage recalls as well the Fogg Behavior Model [18]: once the trigger occurs the action happens if there is enough motivation and ability. 
The reward phase, considers how to reward the user for the action just done in the previous stage. Tree types of rewards are mentioned: reward of the Hunt materialized in resources and goods as money, food, etc.; reward of the Tribe which is given by another user through social-likes, comments, ratings, reviews; reward of the Self which is represented by personal gratification for being consistent with own ideas, mastering an ability or for completing a particular task.

The last phase is the investment, where specific features create the basis for a further usage of the product. An example can be commenting on a photo of a friend on a social network: when the mentioned friends reply the user will be called back to the application restarting the Hook with a trigger.

The work of Eyal produces design guidelines applicable to persuasive technology, for this reason and we will take advantage of some of those principles in the conceptual design of persuasive events.

\subsection{Influence user}

We can often find influence principles applied in several applications, especially oriented to social media [17, 25, 42, 45]. Cialdini wrote a reference book for Influence on people [8]. His principles have already been applied in the marketing field but also in politics and in oratory. The persuasive principle stated by Cialdini are: reciprocity, Commitment and consistency, Social proof, Authority, Liking, Scarcity.

In social media, influence can be implemented through social media features as "browsing other friends' profiles". An example could be the fact that users can be influenced to try a vegetarian diet just because other friends have recently adopted this vegetarian lifestyle Liking principle. Another example could be posting a social media message as: "Vegetarian from today!" to commit in front of other people the engagement in such kind of diet Commitment and consistency principle.

In the literature review we evidence some of those principles of influence applicable to social events, in order to influence users to change behavior.

\section{FOUR CLASSES OF ROLES: LEVERAGING PERSUASION}

We describe four roles to describe users in nature exploration. Our analysis highlights indeed how two main axes seem to characterize the role of users. The first axis characterizes if users execute single-user tasks (Single-user) or if they use the technology collaboratively (Multi-user). The second axis describes if users produce new resources for the exploration (Producer) or if they just use the ones that are available (Consumer). We can indeed find four main roles for users combining the aforementioned scenarios. For each role we will give a definition with a short example.

Creator (Single-user, Producer). Creators add new content for nature exploration as cartographic resources, environment information or experience tips. They are expert in something and eager to share their experience with others. However, their creation task does not involve other users in principle.

Example: creators of Mhikes itineraries use the Mhikes application to trace a GPS route of a certain itinerary physically walking on it.
While walking they take pictures and other multimedia content creating Points Of Interest (POI) enriched with their own explanations. Once completed, the GPS trace is refined using Mhikes back-office algorithms and once ready, the content is made available to the entire community.

Walker (Single-User, Consumer). Walkers use the contents provided by the technology. They take advantage of the technology to be safely guided along the exploration and they are interested in augmenting the experience with multimedia contents to learn and discover more.

Example: Mhikes users use the application to safely remain on the itinerary. Pop-up messages show photos of crossroads with arrows and voice messages to choose the appropriate path. Once in proximity of a Point Of Interest, dedicated contents are shown to learn about plants, flowers, and other environment aspects.

Leader (Multi-User, Producer). Leaders are users that create and organize activities using technology. They use the technology to achieve their primary objective of exploring nature, and secondly they take advantage of the activity to gather other people such as friends, or other users with common interests or similar profile. They are eager to share through social activities. Jointly, leaders may also share useful resources such as transportation means and/or specific equipment dedicated to explorations (e.g. technical clothes). Example: Mhikes leaders are the users that organize weekend-walks and/or nature-related group activities to experience nature collectively.

Follower (Multi-User, Consumer). Followers are users interested in nature exploration but not sufficiently motivated to create content or to organize nature-related social activities. On the other hand, invited by a leader or a friend, they are happy to participate in such kind of activities. They are possibly lacking motivation in organizing, but this is not reflected by a negative mood during the activities. On the contrary, they are enthusiastic of sharing social activities and thankful to the organizers.

Example: In Mhikes, community features are under development and followers are actually a simple group of walkers. Similar to social media, in the future, followers will have access to features that will facilitate connections with leaders. They will be then able to follow the leaders for a walk in the nature, in an exploration, or in other kind of already organized activities.

\section{KEEPING MOTIVATED: A NEW ROLE FOR USERS}

The primary goal of this study is to use HCI to maintain user's motivation for nature exploration through user interaction. Implementing the distinction between the four roles may not prevent users from loosing interest in exploration: repeating the same tasks associated to a given role, may not be interesting. For this reason, it is necessary to give users new perspectives and consequently, making their role in the technology evolve.

Users that want to try exploration for the first time have different needs compared to expert ones. The beginners may just want to be safely guided and to receive information along the exploration. The expert ones may "need more" as for example, creating their 
own itinerary. In both cases, repeating the task without giving a different perspective, may lead not only to loosing interest in using the application, but potentially to losing interest in the whole exploration activity.

We propose to embed features in the HCI technology that persuade users to switch roles, for example, passing from a walker profile to a creator and conversely. The same change can also happen between the other roles. For example, followers, used to waiting for organized activities, may decide to change their approach organizing their own explorations. In the following we will discuss the factors and the persuasive means that may convince the user to change role.

\subsection{The experience factor}

We have mentioned that experience matters in deciding to switch roles. We underline that experience is related not only to the knowhow in exploration, but also to the knowledge of the environment. For example, an expert, for a given location she/he knows well, may be a producer. Conversely, while traveling in a new environment, this expert may endorse the role of follower, joining an exploration group.

\subsection{The similarity factor}

Localization does not impact only on experience but it is also a form of similarity between users. Similarities can relate to common interests, shared background or common friends. Walkers may find out that content dedicated to their interests is missing in the available activities. This may lead them to switch roles, becoming a producer of such content or a leader of a group of exploration enacting a collaborative group-working.

\subsection{How to leverage the switch}

Research in HCI associated to persuasive technology has shown plenty of techniques to trigger, induce and support a behavior change. For example, consumers may be persuaded in becoming creators or leaders, with the aim of being rewarded by other consumers. Reward can be based on social feedback (provided by friends or by community members) or simply on a self reward (successfully reaching a targeted goal) [12].

The moment in which the change should happen, may also depend on intrinsic variables related to the users (motivation and ability) [18]. For example, proposing a consumer to become a producer demands a level of ability that a beginner may not have, and a failure may lead to frustration. For this reason, before suggesting users the possibility of a role-switch it is necessary to investigate what is the context in which such kind of notifications are more effective.

\section{PERSUASIVE EVENTS: PROPERTIES}

\subsection{Definition}

Dictionaries provide several definitions for the word "event": "an occurrence", "something that happens or takes place". For example, the one taken from the Oxford Dictionary, "a planned public or social occasion", gives several interesting perspectives to be discussed. Let us analyze the latter definition. First, the word "planned" suggests a spatial and/or temporal context, often characterized by date, time and GPS position on social media. "Public or social" clarify the privacy issues of an event: it immediately suggests that people are involved with different roles. Finally, the word "occasion" (from latin $o b$-cadere = "something going to fall") demands a clear resolution on what is going to happen.

\subsection{Events on social media}

Among the provided social media features, major social platforms on the Internet ${ }^{1234}$ have a dedicated event system. Having a rich and representative event system, we chose the BlaBlaCar ${ }^{5}$ platform to illustrate events on social media in the following. This platform allows their users to share vehicles to reduce the trip costs, to rate rides and to comment on users' profiles. Also, it includes basic facilities. In this platform, events are shared rides: users can create rides to be shared and/or participate in a ride.

In order to characterize events on social media, we adopted the following methodology for each considered platform: visiting and subscribing to the platform, collecting the details and the possible configuration offered by each system, and finally grouping similar features. From our analysis, the main common features of social media events are: location characterization, scheduling characterization, privacy settings, general personalization of the event, and role of people associated to event.

Location characterization represents where the event happens. It is possible to find two different characterizations: a unique location ${ }^{1,2,3,4}$; or multiple locations associated with a path including a starting location and the ending location ${ }^{5}$. This latter highlights dynamic nature of an event in space: participants can be part of or leave the event in any location. In some platforms ${ }^{3,2}$, the location of an event may be purely virtual in the digital world.

Scheduling characterization represents when the event happens. All the event systems ${ }^{1,2,3,4}$ consider a beginning in time and, eventually, a duration. Similarly to the location characterization, an event may be unique in time or may evolve over time (e.g. dynamic nature in time). For instance, with the BlaBlaCar platform ${ }^{5}$, the timing can vary according to the moment a participant takes part in the event. Another characteristic is given by the possibility to specify a calendar of events referring to the same topic, a feature we have found just on one of the examined platforms ${ }^{4}$.

Privacy settings are related to rights the event organizer gives to the event participants and to users that query the event system. For example, an event can be private ${ }^{1,3}$ : only invited users or the ones that have the password ${ }^{3}$ are allowed to participate in the event. In the event page, users are able to post/comment on a wall ${ }^{1,5}$, to see the list of other participants ${ }^{1,5}$ and, if allowed by the owner, to invite other users ${ }^{1}$.

Event personalization involves several parameters to complete the event. Those parameters are used as additional information for the participants (e.g. event name, description, event organizer name/profile) or simply for decorative purposes (e.g.. personalize the image of the event). Some platforms ${ }^{3}$ have predefined types of

\footnotetext{
${ }^{1}$ https://www.facebook.com/events

${ }^{2}$ https://plus.google.com/events

${ }^{3}$ https://www.eventbrite.com/create

${ }^{4}$ https://www.meetup.com/

${ }^{5}$ https://www.blablacar.co.uk/
} 
event (e.g. party, conference, tournament...) and predefined topics (e.g. music, health, science...) selectable by the event organizer. In some cases ${ }^{3,5}$, it is possible to specify the price, to sell event tickets $^{1,3}$, to explicitly report if the event is free ${ }^{1,3}$ and/or if the event is suitable for a child ${ }^{1}$. In some cases, it is possible to specify the maximum number of participants that can participate in the event $^{2}$ (which in some cases ${ }^{5}$ may be limited).

Participant's role: one of the four roles we identified: walker, creator, leader, follower.

This analysis of event systems in different social network platforms and the identification of event's characteristics serve as a basis to uncover the characteristics of persuasive events.

\subsection{Persuasive properties of events}

From a persuasion perspective, events on social media have interesting characteristics to leverage persuasion in social-based and multi-user interactive systems. Let us analyze these properties in light of persuasion.

Considering Cialdini's principles[8], events may be used to convey influence. Indeed, an event has a group dimension as it implies participants and organizers. Therefore, being invited to take part and taking part in an event is proven to have positive results [8]: it is related to social proof (e.g. "public or social") and commitment. The principle of Social acceptance/rejection states that "people are motivated to do things that win them social acceptance" [18]. In this optic accepting an invitation can figure as the opportunity to do something to confirm a membership (e.g. going to a tribute day). It also gives space to persuade people using events: for example, participating in an event because a friend is participating (liking principle), accepting to go to an event to return a favour (reciprocity principle), or accepting to go to an event because you follow the advice of someone more expert (authority principle). Persuasion offers several techniques oriented to social interactions that can be used in persuasive events (e.g. social support [34]) or that come from the reward/operand conditioning theories tribe reward [12]). In terms of role, being an organizer constitutes a means to support investment [12].

Event's properties also echo with Fogg's behavior model. Temporal planning of events supports one of the fundamental steps in habit forming where the users try to create their own everyday routines to embed change in their lives (e.g. "tiny habits" [18]) The spatial facet (e.g. location) also plays an important role for the context of persuasion. Persuasion have to adapt to context to be effective and location offers several perspectives for this purpose. For example, proximity can be a facilitator while user's favorite locations can be used to respect the user routine principle [18] Thus, an event may be used to increase ability as its facilitating role (e.g. time or money for BlaBlaCar platform). It may also serve as a signal to trigger actions (e.g. notifications).

\section{CONCEPTUAL DESIGN OF PERSUASIVE EVENTS: ILLUSTRATIVE APPROACH}

Having characterized persuasive events, we illustrate their conceptual design, based on a systematic literature review of theoretical and practical principles that have been successfully applied to persuasion. Several approaches for a systematic literature review have

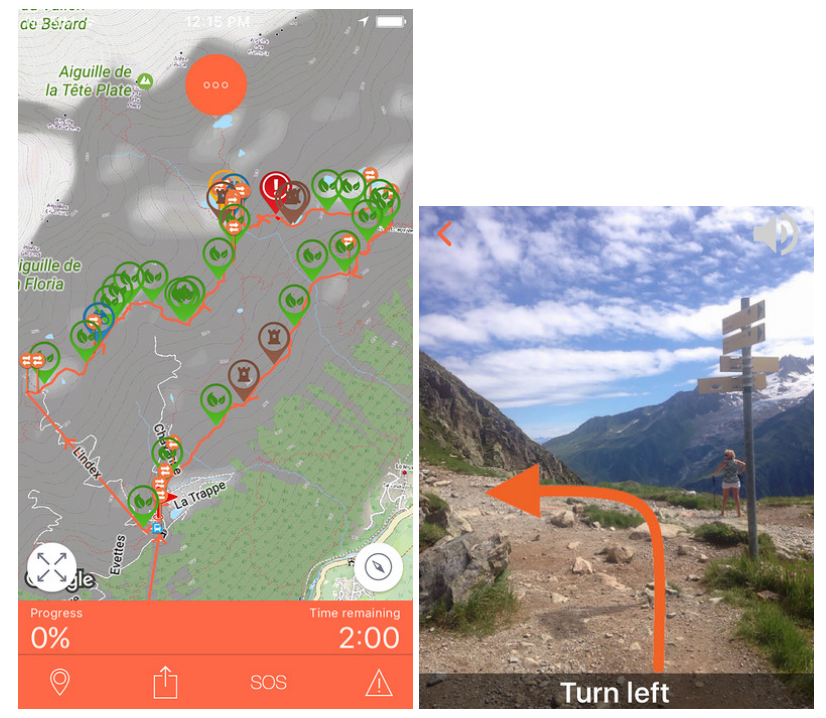

Figure 1: Screenshots of Mhikes application

been used for research purposes, and among them we have chosen to combine two approaches: the one used by Greenberg et al. [23] and the ProSPer framework [15] framing the work on the transtheoretical model of behavior change.

Several works were dedicated to walking/running for example, the application of Rosner et al. [37] that generates walking routes based on digital sketches of users, the studies on pedometers and fitness-oriented systems [2, 4], or on sensing devices for sport [20] whose data have often been processed using machine learning techniques to facilitate the journaling of achieved activities [9].

We will illustrate the examples on an existing platform called Mhikes, designed for the walking/exploring domain. The Mhikes platform ${ }^{6}$ is a free mobile application for Android and iOS, designed to guide users during excursions, hikes and other different type of tours (Figure 1): on the mountains, in the city centers and/or in touristic locations. The Mhikes platform disposes of a database of different itineraries that users can download. The system provides on the route, along with practical directions (e.g."turn right!", "go straight!"), pieces of information about the point of interests associated with the itinerary (e.g. "This mountain is called...", "The history of this place is...").

Mhikes users' profiles are oriented to social media and community features, permitting them to communicate with messages and to share content. Users are divided into Creators (users that create itineraries) and traditional users (who use the system to be guided). Users can rate and share creators' itineraries providing the basic social media features. Even Mhikes does not include any persuasive features, for each stage of the transtheoretical model we have drawn some frame boxes to show how those features could be designed.

\footnotetext{
${ }^{6}$ www.mhikes.com
} 


\section{Pre-Contemplation}

Context: In this stage it is important to "plant the seed", once planted, precontemplators often need time to let them germinate [31]. In this stage, there are no predefined contexts to drive persuasion. We have to consider that in this stage both motivation and ability are low. Motivation in particular can be enhanced by social acceptance while user ability can be in part influenced by the event description, if providing information that the user did not know before.

Example on Mhikes: An example of application on Mhikes is introducing the creation of events associated to Mhikes itineraries. The created event is shared on social media using the basic messaging features, using challenging message. This can be seen as an external trigger that brings the user to discover the platform to support the behavior change.

fim is a 38 years old male married with two children. He is a consultant and has sedentary job occupying large part of his day. Once back home he is used to staying with his family. He does not use any behavior change support system. Fim does not intend to change his behavior: he has his concept of "healthy living" but is not aware of other physical activities apart from gym which he does not consider to be attractive. Fim browsing his usual social network receives an invitation from Mark. The invitation reports the following title:

Invitation
You have been challenged by @Mark for the
\#10kStepsChallenge in@Washington Park!
Discover more

fim does not know that the event is about health/well being, but decides to "discover more" because he is attracted by the challenging undertone of the title, by the proximity of the location (i.e. faciliator) and above all by the fact that the invite was sent by his friend Mark (i.e. social acceptance, reciprocity). He clicks on the content being redirected to the Mhikes website where he can read the event description of the associated itinerary discovering that the minimum recommended number of steps per day for a healthy life is 10000. This has just been means of awareness for fim and a first step toward the contemplation stage.

Analysis: Pre-contemplators by definition are not contemplating the change. For example, they may not be aware or interested in improving their health or saving energy. On the other hand, presenting this topics under a different form, as a game for example, may result to be more attractive for them. For instance, the ice bucket challenge was created for a campaign to raise awareness and support for people with amyotrophic lateral sclerosis (ALS) widely recognized to have gone socially viral [33]. The ice bucket challenge attracted users not familiar with ALS informing them of the problem using a gamification under the form of challenge. This viral phenomenon can be conveyed using the technique of gamifimication consisting of inserting game design elements (challenge a user, gaining points, winning match, collecting badges) in non-gaming contexts $[10,30]$. This viral/challenging side of an event can persuade them to join it since as Fogg remarks, people are motivated to do things that win them social acceptance [18].

\section{Contemplation}

Context: Motivation and ability are supposed to grow in this phase. Social networking involves the explicit modeling of connections between people, which in turn enables and facilitates collaboration [13]. Context in this stage is influenced by those connections between people, and in particular by the similarity of the profiles or by the shared background. Similarity can be based on several criteria: we have mentioned common friends, but also other criteria can be considered, such as geolocalization [46].

Example on Mhikes: Associating an event to an Mhikes itinerary enriches the itinerary with new information that may persuade the user to try the change. An example of this can be discovering friends in the list of participants.

Thanks to the persuasive event fim is now aware of an alternative way to get healthy without going to the gym: participating in events associated with Mhikes itineraries. However, he does not know anything about walking, hiking or running and even if interested, he is not sure that this kind of activity can be adapted for him.

\#10kStepsChallenge 22 participants

@Mark - Friend, you have 5 common friends @fames - Friend, you have a common friend @Susan - You have 2 common friends @Mary

Show more participants

Jim was not sure to be able to join the event associated with the 10000 steps Mhikes itinerary. However, he discovers that the same event has been joined by other friends with a similar lifestyle and background. This information motivates fim to ask these friends about the event and starts considering the idea of participating.

Analysis: In this phase we can use similarity between participants of an event to tip the balance in favor of change to attract the user. Contemplators may think "not feeling competent" [38] and "not believing it will yield a desired outcome" [40]. However, looking at event participants users may figure out that other people they know (with similar background) have actually begun the same behavior change and so they may start considering that they could be able to do the same. Psychology research has shown that we perceive people who are similar to us as credible sources [7, 47]. Cialdini [8] has also shown how people can be influenced by others they like, according to the influence principle called "liking" applicable to this case. The persuasive events highlight, in the participants list, that similar users (or friends) are participating in the event. The persuasive event system indeed suggests first to the user the events that involve common friends to facilitate joining.

\section{Preparation}

Context: In this stage users have decided to start changing; they have their reasons and motivators, but are missing the plan to pass to the action phase. In motivational control theory, Hyland [24] for example, distinguished a mountain climber who chooses a more difficult ascent of a peak because meeting a challenge is a prominent goal from a tourist who chooses the easiest route because the view is the goal [1]. 
Example on Mhikes: On Mhikes different types of itineraries are available with different difficulty levels. Other itineraries offers content connected to wellness as meditation itineraries. The proposed solution can be applied allowing the user to create a series of events which can be named persuasive path.

fim creates a new path selecting as first event "Walking in the city park" (for beginner) then as successor "Discovering Meditation" (for beginner) organized by yoga teachers and as a third one "Walking in the forest" (for intermediate). Once done he invites his friends to the path.

New Path
1. "Walking in the city park" (beginner)
2. "Discover meditation" (beginner)
3. "Walking in the forest" (intermediate)
Add a linkable event

Analysis: A possible solution to the problem that users are missing a plan, is given by introducing in events open-ended design, enabling creativity in self-expression, and promoting awareness and reflection on patterns in health decisions [3]. This design approach permits users to build personalized solutions to their own needs without relying on pre-configured ones. For example, in order to achieve the process of change, the user is allowed to choose or specify a temporal sequence of events (i.e. path of events). Such a sequence is then mapped onto the phases of the TTM in order to follow the stage of changes [35].

\section{Action}

Context: According to Fogg, users in the action phase are highly motivated and able to perform the change. They have received a trigger to commit into the first concrete step of change. For a runner for example, it can be represented by taking the first jogging path. In this moment users are putting in practice the behavior and they need to know if they are doing it correctly.

Example on Mhikes: Mhikes already offers the basic features to share contents on social media. Persuasion can be integrated allowing the event system to suggest users to create new contents as photos, videos or reviews to be shared among users. A reward by the community can be provided using gamification.

fim has begun the change, he has participated to his first "Walking event" in the city park and he liked it. He is currently motivated to repeat the experience.

During the last event "Walking in the City Park" fim was asked by the persuasive event system to take pictures and to share them with his friends. At the end of the event fim was asked by the system to rate the event to provide new information to the possibly useful for other users of Mhikes's community.

\section{Share your experience}

Tell your friends about your experience in Walking in the City Park and earn experience point for your Mhikes profile.

$$
\begin{array}{|l|}
\hline \text { Take a picture and share it } \\
\hline \hline \text { Send this itinerary to a Friend } \\
\text { (5 Xp. points) }
\end{array}
$$

Write a review of this itinerary (10 Xp. points)

fim is happy to see that his friends commented on his pictures and that his rating has been considered useful by users. This made him gain two new badges "Event Photographer" and "Newbie Reviewer" making him satisfied. Fim is now motivate to check what he should do to collect other badges..

Analysis: The motivation of a user can increase when starting the change but is not guaranteed to last over a long period. In the action phase, motivation is high and the persuasive system has to persuade users to create new trigger elements to be used when motivation fades. Examples can be found in the theories of Skinner [41], through mechanisms involving the awarding of points or tokens to reinforce the behavior [43]. Reward can be combined with social gamification to avoid people falling off from achieving their goals [19]. The social aspect of self monitoring in fact,according to Tosco[44] additionally helps to staying motivated.

This strategy is similar to what is called "investment/internal trigger" by Eyal. In his Hook model the user in the action phase does "a bit of work" to cause a further trigger [12]. Those strategies can be used to build personalized triggers that turns into routines, habits and mechanisms in the everyday life. Creating contents such as videos or pictures on social media can be seen as the "bit of work" caused by the high motivation of the user. When another one comments those contents a notification is provided to the author. We have to consider that statistics on "how many people have found the rating useful" or the number of comments, likes, re-blog, or share, are a form of social reward. They are used in persuasive event to create badges in the user profile (e.g."10 pictures collected","3 useful ratings"). This is motivating users to publish more contents, reply to comments and so to remember the change they want to achieve. Rating an event is moreover a form of social collaboration, since it contributes to build a social shared knowledge between users. This aspect has been found successful and viral in other problematic as the research of a "good" restaurant. Communities as TripAdvisor ${ }^{7}$, Yelp $^{8}$, ForSquare ${ }^{9}$ have become reference applications for the food topic.

\section{Maintenance}

Context: Users in this stage are struggling to prevent falling in a relapse phase [31] but some point, behaviors become sustained over time and integrated into their lifestyle so that the individual can leave the cycle of change[31]. Even if notifications in HCI are used to trigger users to act, in the maintenance phase users have already passed to the action participating to several persuasive events. In this case, notifying the same message to trigger the action can result in repetitive and not attractive anymore [15].

Example on Mhikes: Mhikes does not include a persuasive notification system at the moment. A possible implementation could integrate the delayed notification approach: when the users remember to perform the action they are rewarded with special

\footnotetext{
${ }^{7}$ https://www.tripadvisor.com/

${ }^{8}$ https://www.yelp.com/

${ }^{9}$ https://foursquare.com/
} 
points named "straight points" that are provided just for continuous actions and subtracted when the action is not performed.

fim has discovered walking, he has created a new network of friends around this activity and wants to integrate it permanently in his life.

It's Friday afternoon and Jim has just walked out from his office. While headed home (context) he checked on Mhikes the list available events for the Weekend (action). Mhikes recorded this pattern two times and created a rule in the system. Now when fim leaves the office, if he checks the available events on Mhikes he gains one "straight point".

Your strength has grown to 20 (+5 Sp. Points)

Congratulation Jim, we hare happy to see that you do not want to miss Mhikes's activities in this weekend. We hope you will enjoy the available events!

Check events for the Week End

In case this action of checking events does not happen, once home Mhikes informs him with a (delayed) notification about available events for the weekend. In this case he will not collect 5 points but just one.

\section{Mhikes events for the weekend}

Hello Jim, we hope to see you participating to some Mhikes events in the weekend. Check the available ones on Mhikes and reach 19 point of strength $(+1 \mathrm{Sp}$. Point)!

Check events for the Week End

Analysis: Many controversial studies have discussed the problem questioning if persuasion is sustainable or not [5] [26]. Fogg has theorized the concept of "tiny habit" in his behavior model [18]. The strategy is to associate to a situation the action, so that when users found themselves in that situation, they are triggered to repeat the associate action [18]. This approach can be used to create habits to continue doing the change in the maintenance phase. In this context we propose the concept of delayed notifications associated with a reward in the persuasive strategy to implement the "tiny habit" approach for persuasive events. In persuasive events for maintenance phase we rethink the concept of notifying to introduce "delayed notification". When the persuasive system detects a context in which a notification should be triggered (e.g. Friday afternoon) waits for the user to do the action connected with the behavior (e.g. search for an event for Saturday morning). If users have developed the habit, they will perform the action and they will be rewarded for this autonomous action. Otherwise a delayed notification will be triggered to avoid loosing the opportunity of performing the behavior and to enforce the habit-forming. This may lead to go back to a previous stage of TTM.

\subsection{Conclusion and Future Work}

In this work, we have evidenced how HCI solutions for exploration usually implement implicit roles in their technology. We characterized four classes of roles that users may endorse, according to two axes: a single/multi vs. consumer/producer usage, giving concrete examples. Finally, we discussed why users should switch their role over time, underlining some factors and approaches that may lead to this change. We also provided a general overview of current event systems on social media deriving a general definition of event along with related properties. Then, we have shown how a link between the concept of event and behavior change can be enhanced using social media. Our literature review, instantiated on the transtheoretical model, gives concrete solutions of this approach through scenario-based examples on the existing platform Mhikes.

We are working to prototype the persuasive features on the aforementioned platform Mhikes, where the implementation will enrich our vision with concrete feedback coming from user experiences. We pan to refine our persuasive features with future findings to produce a structuring framework supporting not only the development but also the evaluation of persuasive events for social media.

\section{ACKNOWLEDGMENTS}

The authors would like to thank Easy Mountain for their partnership on this project.

\section{REFERENCES}

[1] James T Austin and Jeffrey B Vancouver. 1996. Goal constructs in psychology: Structure, process, and content. Psychological bulletin 120, 3 (1996), 338.

[2] Graham Baker, Nanette Mutrie, and Ruth Lowry. 2008. Using pedometers as motivational tools: Are goals set in steps more effective than goals set in minutes for increasing walking? International fournal of Health Promotion and Education 46, 1 (2008), 21-26.

[3] Eric PS Baumer, Sherri Jean Katz, Jill E Freeman, Phil Adams, Amy L Gonzales, John Pollak, Daniela Retelny, Jeff Niederdeppe, Christine M Olson, and Geri K Gay. 2012. Prescriptive persuasion and open-ended social awareness: expanding the design space of mobile health. In Proceedings of the ACM 2012 conference on Computer Supported Cooperative Work. ACM, 475-484.

[4] Dena M Bravata, Crystal Smith-Spangler, Vandana Sundaram, Allison L Gienger, Nancy Lin, Robyn Lewis, Christopher D Stave, Ingram Olkin, and John R Sirard. 2007. Using pedometers to increase physical activity and improve health: a systematic review. Fama 298, 19 (2007), 2296-2304.

[5] Hronn Brynjarsdottir, Maria Håkansson, James Pierce, Eric Baumer, Carl DiSalvo, and Phoebe Sengers. 2012. Sustainably unpersuaded: how persuasion narrows our vision of sustainability. In Proceedings of the SIGCHI Conference on Human Factors in Computing Systems. ACM, 947-956.

[6] Keith William John Cheverst, Ian Norman Gregory, and Helen Turner. 2016. Encouraging Visitor Engagement and Reflection with the Landscape of the English Lake District: Exploring the potential of Locative Media. In International Workshop on'Unobtrusive User Experiences with Technology in Nature'. 1-5.

[7] Robert B Cialdini. 2001. Science and practice. (2001).

[8] Robert B Cialdini and Nathalie Garde. 1987. Influence. Vol. 3. A. Michel.

[9] Sunny Consolvo, David W McDonald, Tammy Toscos, Mike Y Chen, Jon Froehlich, Beverly Harrison, Predrag Klasnja, Anthony LaMarca, Louis LeGrand, Ryan Libby, et al. 2008. Activity sensing in the wild: a field trial of ubifit garden. In Proceedings of the SIGCHI Conference on Human Factors in Computing Systems. ACM, 17971806.

[10] Sebastian Deterding, Miguel Sicart, Lennart Nacke, Kenton O'Hara, and Dan Dixon. 2011. Gamification. using game-design elements in non-gaming contexts. In CHI'11 Extended Abstracts on Human Factors in Computing Systems. ACM, 2425-2428.

[11] Carlo C DiClemente, James O Prochaska, Scott K Fairhurst, Wayne F Velicer, Mary M Velasquez, and Joseph S Rossi. 1991. The process of smoking cessation: an analysis of precontemplation, contemplation, and preparation stages of change. fournal of consulting and clinical psychology 59, 2 (1991), 295.

[12] Nir Eyal. 2014. Hooked: How to build habit-forming products. Penguin.

[13] Gunther Eysenbach. 2008. Medicine 2.0: social networking, collaboration, participation, apomediation, and openness. Journal of medical Internet research 10, 3 (2008), e22.

[14] Alessandro Fenicio and Gaëlle Calvary. 2015. Persuasion Through an Ambient Device: Proof of Concept and Early Evaluation of CRegrette, a Smoking Cessation System. In European Conference on Ambient Intelligence. Springer, 252-267.

[15] Alessandro Fenicio, Gaëlle Calvary, Yann Laurillau, and Jean Vanderdonckt. 2016. ProSPer: a MOST model extension applied to persuasive interactive system. In Proceedings of British HCI 2016 Conference (HCI 2016). 
[16] BJ Fogg. 2002. Persuasive Technology: Using Computers to Change What We Think and Do (Interactive Technologies). (2002).

[17] BJ Fogg and Daisuke Iizawa. 2008. Online persuasion in Facebook and Mixi: a cross-cultural comparison. In Persuasive technology. Springer, 35-46.

[18] Brian J Fogg. 2009. A behavior model for persuasive design. In Proceedings of the 4th international Conference on Persuasive Technology. ACM, 40.

[19] Brain J Fogg. 2009. Creating persuasive technologies: an eight-step design process.. In PERSUASIVE. 44.

[20] Thomas Fritz, Elaine M Huang, Gail C Murphy, and Thomas Zimmermann. 2014 Persuasive technology in the real world: a study of long-term use of activity sensing devices for fitness. In Proceedings of the SIGCHI Conference on Human Factors in Computing Systems. ACM, 487-496.

[21] Geoffrey W Greene, Susan R Rossi, Gabrielle Richards Reed, Cynthia Willey, and James O Prochaska. 1994. Stages of change for reducing dietary fat to $30 \%$ of energy or less. Fournal of the American Dietetic Association 94, 10 (1994), $1105-1112$.

[22] Andrea Grimes and Rebecca E Grinter. 2007. Designing persuasion: Health technology for low-income African American communities. In International Conference on Persuasive Technology. Springer, 24-35.

[23] Helen Ai He, Saul Greenberg, and Elaine M Huang. 2010. One size does not fit all: applying the transtheoretical model to energy feedback technology design. In Proceedings of the SIGCHI Conference on Human Factors in Computing Systems. ACM, 927-936.

[24] Michael E Hyland. 1988. Motivational control theory: An integrative framework. Fournal of Personality and Social Psychology 55, 4 (1988), 642.

[25] Noreen Kamal and Sidney Fels. 2012. Determining the determinants of health behaviour change through an online social network. In Persuasive Technology. Design for Health and Safety. Springer, 1-12.

[26] Bran Knowles, Lynne Blair, Stuart Walker, Paul Coulton, Lisa Thomas, and Louise Mullagh. 2014. Patterns of persuasion for sustainability. In Proceedings of the 2014 conference on Designing interactive systems. ACM, 1035-1044.

[27] Tuomas Lehto and Harri Oinas-Kukkonen. 2011. Persuasive features in webbased alcohol and smoking interventions: a systematic review of the literature. fournal of medical Internet research 13, 3 (2011), e46.

[28] Bess H Marcus, Stephen W Banspach, R Craig Lefebvre, Joseph S Rossi, Richard A Carleton, and David B Abrams. 1992. Using the stages of change model to increase the adoption of physical activity among community participants. American journal of health promotion 6, 6 (1992), 424-429.

[29] Simon J Marshall and Stuart JH Biddle. 2001. The transtheoretical model of behavior change: a meta-analysis of applications to physical activity and exercise. Annals of behavioral medicine 23, 4 (2001), 229-246.

[30] Jane McGonigal. 2011. Reality is broken: Why games make us better and how they can change the world. Penguin.

[31] WR Miller and S Rollnick. 2002. Motivational interviewing: Preparing people for change Guilford. New York (2002).

[32] Sosuke Miura, Pamela Ravasi, and Masanori Sugimoto. 2008. Nurturing Learners' Communities by Creating and Sharing Maps. PsychNology fournal 6, 3 (2008), 269-289.

[33] Michael Y Ni, Brandford HY Chan, Gabriel M Leung, Eric HY Lau, and Herbert Pang. 2014. Transmissibility of the Ice Bucket Challenge among globally influential celebrities: retrospective cohort study. Bmj 349 (2014), g7185.

[34] Harri Oinas-Kukkonen and Marja Harjumaa. 2008. A systematic framework for designing and evaluating persuasive systems. In International Conference on Persuasive Technology. Springer, 164-176.

[35] James O Prochaska and Wayne F Velicer. 1997. The transtheoretical model of health behavior change. American journal of health promotion 12, 1 (1997), 38-48.

[36] Yvonne Rogers, Sara Price, Geraldine Fitzpatrick, Rowanne Fleck, Eric Harris, Hilary Smith, Cliff Randell, Henk Muller, Claire O’Malley, Danae Stanton, et al 2004. Ambient wood: designing new forms of digital augmentation for learning outdoors. In Proceedings of the 2004 conference on Interaction design and children: building a community. ACM, 3-10.

[37] Daniela K Rosner, Hidekazu Saegusa, Jeremy Friedland, and Allison Chambliss. 2015. Walking by Drawing. In Proceedings of the 33rd Annual ACM Conference on Human Factors in Computing Systems. ACM, 397-406.

[38] Richard M Ryan and Edward L Deci. 2000. Intrinsic and extrinsic motivations: Classic definitions and new directions. Contemporary educational psychology 25 1 (2000), 54-67.

[39] Kimiko Ryokai and Alice Agogino. 2013. Off the paved paths: Exploring nature with a mobile augmented reality learning tool. International fournal of Mobile Human Computer Interaction (IFMHCI) 5, 2 (2013), 21-49.

[40] Martin EP Seligman. 1975. Helplessness: On depression, development, and death WH Freeman/Times Books/Henry Holt \& Co.

[41] Burrhus Frederic Skinner. 1953. Science and human behavior. Simon and Schuster.

[42] Agnis Stibe and Harri Oinas-Kukkonen. 2012. Comparative analysis of recognition and competition as features of social influence using twitter. In Persuasive Technology. Design for Health and Safety. Springer, 274-279.

[43] Martin Sundel and Sandra S Sundel. 2004. Behavior change in the human services: Behavioral and cognitive principles and applications. Sage.
[44] Tammy Toscos, Anne Faber, Kay Connelly, and Adity Mutsuddi Upoma. 2008. Encouraging physical activity in teens Can technology help reduce barriers to physical activity in adolescent girls? In Pervasive Computing Technologies for Healthcare, 2008. PervasiveHealth 2008. Second International Conference on. IEEE, 218-221.

[45] Thijs Waardenburg, Robbert Winkel, and Maarten H Lamers. 2012. Normative social influence in persuasive technology: intensity versus effectiveness. In Persuasive Technology. Design for Health and Safety. Springer, 145-156.

[46] Yu Zheng, Xing Xie, and Wei-Ying Ma. 2008. Determining user similarities based on location histories. (Nov. 3 2008). US Patent App. 12/264,038.

[47] Philip G Zimbardo and Michael R Leippe. 1991. The psychology of attitude change and social influence. Mcgraw-Hill Book Company. 